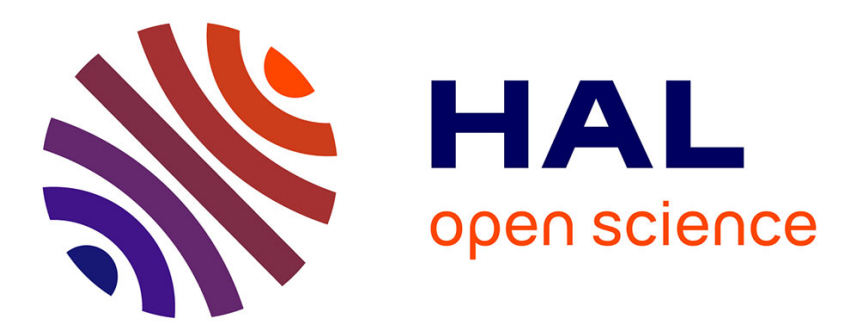

\title{
Three-wavelength broadband soot pyrometry technique for axisymmetric flames
}

Juan José Cruz, Felipe Escudero, Emilio Álvarez, Luís Fernando Figueira da Silva, Gonzalo Carvajal, María Thomsen, Andrés Fuentes

\section{To cite this version:}

Juan José Cruz, Felipe Escudero, Emilio Álvarez, Luís Fernando Figueira da Silva, Gonzalo Carvajal, et al.. Three-wavelength broadband soot pyrometry technique for axisymmetric flames. Optics Letters, 2021, 46 (11), pp.2654. 10.1364/OL.424529 . hal-03313673

\section{HAL Id: hal-03313673 \\ https://hal.science/hal-03313673}

Submitted on 6 Oct 2021

HAL is a multi-disciplinary open access archive for the deposit and dissemination of scientific research documents, whether they are published or not. The documents may come from teaching and research institutions in France or abroad, or from public or private research centers.
L'archive ouverte pluridisciplinaire HAL, est destinée au dépôt et à la diffusion de documents scientifiques de niveau recherche, publiés ou non, émanant des établissements d'enseignement et de recherche français ou étrangers, des laboratoires publics ou privés. 


\title{
Three wavelengths broadband soot pyrometry technique for axisymmetric flames
}

\author{
Juan José Cruz ${ }^{1}$, Felipe Escudero ${ }^{1}$, Emilio Álvarez ${ }^{1}$, Luís Fernando Figueira da Silva ${ }^{2,3}$, \\ Gonzalo Carvajal ${ }^{4}$, María Thomsen ${ }^{5}$, And AndrÉs Fuentes ${ }^{1 *}$ \\ ${ }^{1}$ Departamento de Industrias, Universidad Técnica Federico Santa María, Av. España 1680, Valparaíso, Chile \\ ${ }^{2}$ Department of Mechanical Engineering, PUC-Rio, R. Marquês de São Vicente, 225, Rio de Janeiro, Brazil \\ ${ }^{3}$ PPRIME UPR3346 CNRS, ENSMA and University of Poitiers, 1 Avenue Clément Ader, Futuroscope Chasseneuil 86961, France \\ ${ }^{4}$ Departamento de Electrónica, Universidad Técnica Federico Santa María, Av. España 1680, Valparaíso, Chile \\ ${ }^{5}$ Faculty of Engineering and Sciences, Universidad Adolfo Ibáñez, Santiago, Chile \\ *Corresponding author: andres.fuentes@usm.cl
}

Compiled October 6, 2021

\begin{abstract}
Soot temperature measurements in laminar flames are often performed through two-color broadband emission pyrometry (BEMI) or modulated-absorptionemission (BMAE) techniques, using models to relate the ratio between flame intensities at two different wavelengths with soot temperature. To benefit from wider spectral range and increase the accuracy of experimental estimation of soot temperature, this work proposes a new approach that uses three-color broadband images captured with a basic color camera. The methodology is first validated through simulations using numerically generated flames from the CoFlame code, and then used to retrieve soot temperature in an experimental campaign. The experimental results show that using three-color and BEMI provides smoother reconstruction of soot temperature than two-color and BMAE when small disturbances exist in the measured signals due to a reduced experimental noise effect. A sensitivity analysis shows that the retrieved temperature from three-color BEMI is more resilient to variations on the ratio of measured signals than BMAE, which is confirmed by an error propagation analysis based on a Monte Carlo approach. () 2021 Optical Society of America
\end{abstract}

http://dx.doi.org/10.1364/ao.XX.XXXXXX

\section{INTRODUCTION}

Pyrometry for estimating soot temperature $\left(T_{S}\right)$ can be implemented either by alternating narrowband monochromatic filters [1] or using a standard color camera to simultaneously capture broad spectral bands at the Red, Green, and Blue (RGB) channels [2-4]. Flame emission pyrometry techniques (EMI) $[2,4,5]$ normally neglect the self-absorbed thermal radiation along the optical detection path [6]. This assumption simplifies the inversion procedure required to determine $T_{S}$, but it may generate uncertainties in the estimated values $(\leq 50 \mathrm{~K})$ [3]. More- over, EMI techniques require prior knowledge of the soot absorption function $\left(E_{m}\right)$ spectral behavior, which is not yet well understood [7]. In contrast, light modulated absorption/emission (MAE) techniques are used to measure the soot volume fraction $\left(f_{v}\right)$ and $T_{s}$ by carrying out simultaneous measurements of light extinction and flame emission at two narrow spectral bands $(<10 \mathrm{~nm})[8,9]$. The narrow band MAE technique has also been extended to broadband images (BMAE) using a tri-CCD camera as a pyrometer in a non-buoyant flame [10]. However, capturing the emitted signal in a broad spectral band complicates the deconvolution procedure required to retrieve the local soot emission (and thus, $T_{s}$ ) since a spectral integration must be performed. The combination of different wavelength pairs is commonly used in soot pyrometry; however, this procedure may lead to different temperature estimates. Indeed, using emission measurements at three wavelengths could yield three different temperatures, according to the color ratio used $(G / R, B / R$ or $B / G)$, two of which are independent $[11,12]$. In such case, discrepancies are often circumvented by reporting the mean temperature obtained for different color ratios [3]. Thus, it may seem not entirely correct to use the information of only two wavelengths. Although improvements can be expected from combining the three wavelengths from the color channels to generate a single temperature, such a model has not been reported.

This letter presents a novel methodology to retrieve $T_{S}$ employing a single model using three visible broad spectral bands. The methodology is applied to a canonical laminar axisymmetric diffusion flame and tested at three different levels. First, the method is validated using synthetic images obtained from a flame simulated with the CoFlame code [13]. Then, the broadband emission (BEMI) and the BMAE techniques at two and three color ratios (2-BEMI, 2-BMAE, 3-BEMI and 3-BMAE) are used to experimentally retrieve $T_{S}$ and their results are compared. Finally, an error propagation and sensitivity analysis are performed to assess the uncertainties of each three-color method.

\section{THREE COLOR SOOT PYROMETRY MODEL}

The convoluted flame radiative intensity emitted and selfabsorbed by soot particles $\left(I_{\lambda}\right)$ for a given wavelength $(\lambda)$, rep- 
resents the integrated local soot emission (neglecting scattering effects) along the photodetector line-of-sight (l), and can be expressed as $[6,9]$ :

$$
I_{\lambda}\left(l, T_{S}\right)=\int_{l} \kappa_{\lambda}(l) I_{\lambda}^{b b}\left(T_{S}\right) \exp \left[-\int_{l^{\prime}} \kappa_{\lambda}\left(l^{\prime}\right) \mathrm{d} l^{\prime}\right] \mathrm{d} l,
$$

where $\kappa_{\lambda}$ is the soot absorption coefficient derived from $\kappa_{\lambda}=$ $f_{v} 6 \pi E_{m} / \lambda$ (Rayleigh regime). The exponential term represents the self-absorption along the remaining flame path to the photodetector $\left(l^{\prime}\right)$ and $I_{\lambda}^{b b}$ is the blackbody radiative intensity that depends on $T_{S}$, which is assumed to be in thermal equilibrium with the gas-phase. When using an RGB camera, $I_{\lambda}$ is weighted by the sensitivity of detection system $\left(\eta_{\lambda}\right)$ and spectrally integrated in a broadband $i \in\{\mathrm{R}, \mathrm{G}, \mathrm{B}\}$. Thus,

$$
S_{i}^{\prime}=\int_{\lambda_{i}} \eta_{\lambda} I_{\lambda}\left(l, T_{S}\right) \mathrm{d} \lambda
$$

represents the projected flame radiative intensity captured by the sensor of the RGB camera at each broadband.

In the BMAE technique, simultaneous emission and extinction measurements must be carried out, which are typically performed by modulating an external back-light source [10]. During the modulation, the flame radiative intensity (emission) is captured with and without the source, denoted by $S_{i}^{\prime}$ and $S_{i}$, respectively, and then used in the following expression:

$$
\left(\frac{S_{i}-S_{i}^{\prime}}{I_{i}-I_{i}^{\prime}}\right)=\frac{\int_{\lambda_{i}} \phi_{\lambda} \eta_{\lambda} \exp \left[-\int_{l} \kappa_{\lambda}(l) \mathrm{d} l\right] \mathrm{d} \lambda}{\int_{\lambda_{i}} \phi_{\lambda} \eta_{\lambda} \mathrm{d} \lambda},
$$

where $I_{i}$ and $I_{i}^{\prime}$ are images of the back-light source and background intensity, respectively, and $\phi_{\lambda}$ is the spectral backlight radiance. The $f_{v}$ can be obtained by replacing the $\kappa_{\lambda}=$ $f_{v} 6 \pi E_{m} / \lambda$ definition in the right-hand-side (RHS) of Equation 3. The soot absorption function at different wavelengths is represented as $E_{m}(\lambda)=0.284+0.272(\ln (\lambda))^{2}+0.057 \frac{\ln (\lambda)}{\lambda}[14]$. For this purpose, an iterative process is performed to retrieve the $f_{v}$ that matches the RHS model to the left-hand-side (LHS) that represents the experimentally measured transmissivity. Due to the self-absorption term in Eq. 3, the iterative process starts from the outer flame moving towards its central axis [10].

In the BMAE approach, $T_{S}$ is estimated from flame radiative broadband intensity measurements $S_{i}^{\prime}$ (see Fig 1a). Here, the convoluted intensities captured by the RGB camera may be defined as a ratio $\Theta_{3 S}=S_{\mathrm{G}}^{\prime} S_{\mathrm{B}}^{\prime} /\left[S_{\mathrm{R}}^{\prime}\right]^{2}$, yielding:

$$
\Theta_{3 S}=\frac{\int_{\lambda_{G}} \eta_{\lambda} I_{\lambda}\left(l, T_{S}\right) \mathrm{d} \lambda \int_{\lambda_{B}} \eta_{\lambda} I_{\lambda}\left(l, T_{S}\right) \mathrm{d} \lambda}{\left[\int_{\lambda_{R}} \eta_{\lambda} I_{\lambda}\left(l, T_{S}\right) \mathrm{d} \lambda\right]^{2}} .
$$

Once $f_{v}$ is experimentally determined, the only unknown property in the RHS of Eq. 4 is $T_{s}$, which appears in $I_{\lambda}^{b b}$ through the integrated flame radiative intensity $I_{\lambda}$ (see Eq. 1 ). Using a similar approach to the one used to determine $f_{v}$, the RHS model of Eq. 4 is computed for different values of $T_{S}$ until matching the LHS ratio. This procedure is performed for each flame height to obtain a spatial distribution of $T_{S}$ denoted as $T_{S} 3$-BMAE.

The same methodology can be applied for obtaining a $T_{S}$ field by a common two-color ratio ( $T_{S}$ 2-BMAE) by modifying Eq. 4 accordingly [10], employing a two-color ratio $\Theta_{2 S}=S_{\mathrm{G}}^{\prime} / S_{\mathrm{R}}^{\prime}$. In general, the BMAE technique suffers two drawbacks: (1) the spectral dependence of the self-absorption term may lead to different retrieved values for $T_{S}[10]$, and (2) the recovered
$T_{\mathcal{S}}$ is highly susceptible to the convoluted intensity noise affecting the reconstruction of $f_{v}$ during the deconvolution process [15]. In contrast, for the BEMI technique [2], the requirement of prior knowledge of $\kappa_{\lambda}$ is avoided by neglecting the exponential term in Eq. 1. By ignoring the self-absorption in Eq. 2 [16], it is possible to derive a novel color soot pyrometry ratio $\Theta_{3 H}=H_{\mathrm{G}}(l) H_{\mathrm{B}}(l) /\left[H_{\mathrm{R}}(l)\right]^{2}$ based on the combination of three deconvoluted broadband image intensities according to:

$$
\Theta_{3 H}=\frac{\int_{\lambda_{G}} \eta_{\lambda} \frac{E_{m}}{\lambda} I_{\lambda}^{b b}\left(T_{S}\right) \mathrm{d} \lambda \int_{\lambda_{B}} \eta_{\lambda} \frac{E_{m}}{\lambda} I_{\lambda}^{b b}\left(T_{S}\right) \mathrm{d} \lambda}{\left[\int_{\lambda_{R}} \eta_{\lambda} \frac{E_{m}}{\lambda} I_{\lambda}^{b b}\left(T_{S}\right) \mathrm{d} \lambda\right]^{2}}
$$

where $H_{i}(l)$ represents the local soot emission at the symmetry plane obtained by deconvoluting the integrated broadband camera images from $S_{i}^{\prime}=\int_{l} H_{i}(l) \mathrm{d} l$ (see Fig 1a). An onion-peeling discretization coupled with a Tikhonov regularization is used to perform the deconvolution in order to obtain $H_{i}(l)$ for the $\mathrm{R}, \mathrm{G}$ and $\mathrm{B}$ camera channels. The Tikhonov regularization introduces a regularization parameter that helps to stabilize the solution of the original ill-posed deconvolution problem [17]. The BEMI model provides a unique solution for $T_{S}$, denoted as $T_{S}$ 3-BEMI. Indeed, $T_{S}$ is obtained employing an a priori constructed lookup table that relates the experimentally determined ratio $\Theta_{3 H}$ with the RHS of Eq. 5 evaluated at different $T_{s}$. Note that this approach is unfeasible for the BMAE technique since the spectral integration of the self-aborption term precludes the deconvolution of Eq. 4. In an analogous way, two wavelengths ( $T_{S} 2$-BEMI) can be employed by using a modified version of Eq. 5 with $\Theta_{2 H}=H_{\mathrm{G}} / H_{\mathrm{R}}$ [2]. Furthermore, both methodologies can be applied in sooting flames under different combustion regimes, as long as they have an axisymmetric geometry.

In this work, the robustness of the $T_{S} 3$-BEMI and $T_{S} 3$-BMAE is tested through a Monte Carlo (MC) approach by perturbing the experimental data [18]. Additionally, a sensitivity analysis is performed for the 3-BEMI and 3-BMAE techniques (see Supplemental Document for details).

\section{INPUT DATA AND VALIDATION}

Fig. 1a shows a cross section of the flame with the variables involved in the model developed in Section 2. The spectral sensitivities $\left(\eta_{\lambda}\right)$ of the detection system at each broadband channel are shown in Fig. 1b. Note that to construct $\eta_{\lambda}$, the filter transmissivities were measured and the camera manufacturer's response curve was used. Also, the radiometric back-light emission $\phi_{\lambda}$ measured with a calibrated spectrometer. Experimental projected broadband flame emission images are obtained from an axisymmetric canonical laminar diffusion flame established on a Gülder type burner. Constant volumetric flow rates of pure ethylene (0.194 slpm) and coflow air (284 slpm) are imposed. The experimental setup (described in details in the supplemental document) is composed by broadband light sources and a Basler RGB CMOS. To improve the signal-to-noise ratio (SNR) in channel B (usually the channel with the weakest intensity), a combination of visible and IR cut filter were used to reduce the camera sensitivity in the red channel. A SNR above 20 was obtained within the flame according to channel $\mathrm{B}$, and this value was sufficient for the convergence of the algorithm. Radical chemiluminescence detection can be neglected in this work because it occurs in narrow bands of a few nanometers, compared to the strong and continuous spectral emission of soot.

The measured two-color $\left(\Theta_{2 S}\right)$ and three-color $\left(\Theta_{3 S}\right)$ ratios of projected flame radiative intensities used for obtaining $T_{S}$-BMAE 
a)

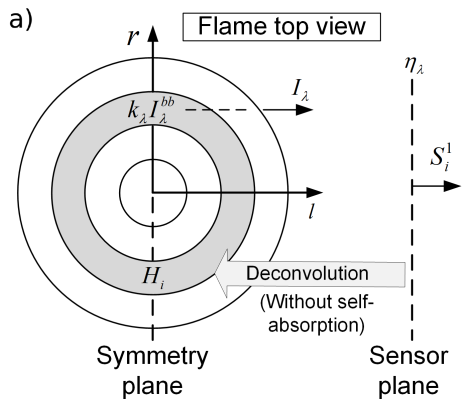

c)

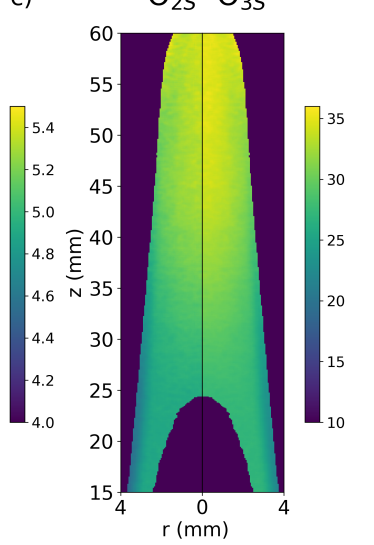

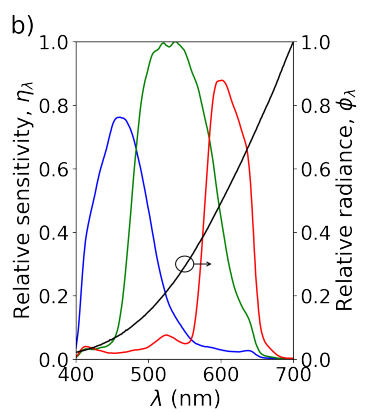

d)

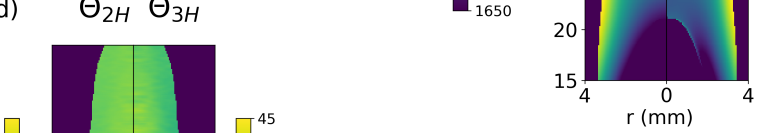

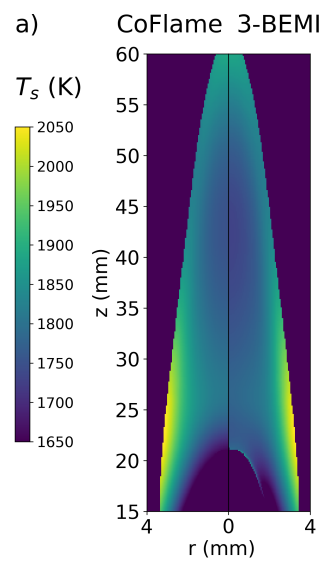

b)

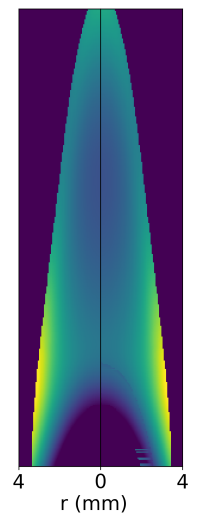

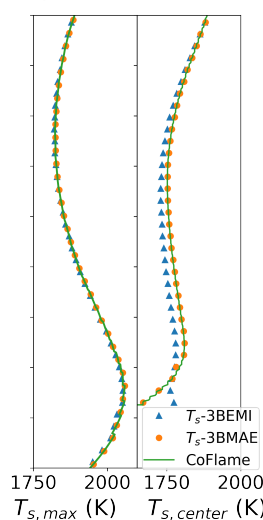

Fig. 2. (a) Numerical $T_{S}$ fields from CoFlame (left-panels) and determined using 3-BEMI and 3-BMAE approaches (rightpanels), and (b) maximum (left) and centerline (right) temperatures for each flame height.

broadband extinction measurements (see Eq. 3). Qualitatively, the four retrieved fields of $T_{s}$ present a high temperature zone $(\sim 1830 \mathrm{~K})$ near the visible flame edge close to the burner exit. Also, a decrease of $T_{S}$ is observed toward the flame centerline, independent of the technique employed. The BEMI approach provides a smoother $T_{S}$ field than the BMAE model because the self-absorption term is neglected. Indeed, this term provides an additional source of noise coming from the deconvolution of $f_{v}$. Moreover, the experimental noise of emission measurements ( $\sim 5 \%$ ) deteriorates the $T_{S}$ reconstruction procedure. This is particularly relevant in the lower region of the flame, where the self-absorption effect is not negligible, leading to a maximum temperature difference of $55 \mathrm{~K}$ between BMAE and BEMI when using three broadbands. This effect also leads to slightly higher temperatures for BMAE than BEMI along the inner flame region. Fig. $3 \mathrm{~b}$ presents the maximum (left) and centerline (right) values of $T_{S}$ (radial profiles are shown in the supplemental document). Comparing these profiles for $T_{S} 3$-BEMI and $T_{S} 3$-BMAE, it can be observed that maximum $T_{S}$ behaves similarly, regardless of the technique employed. Nevertheless, scattered results are observed for the 3-BMAE approach, specially toward the flame
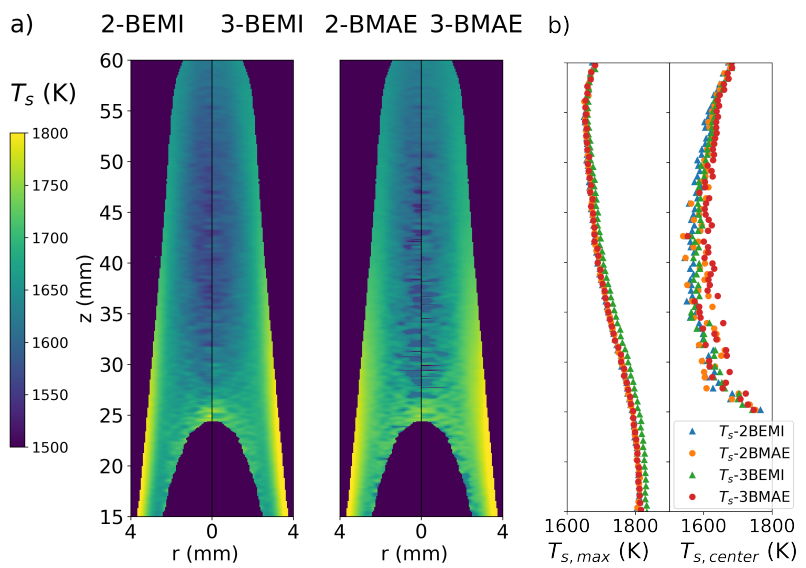

Fig. 3. (a) Temperature fields retrieved with BEMI and BMAE approaches for two and three wavelengths, and (b) maximum (left) and centerline (right) temperature for each flame height. 

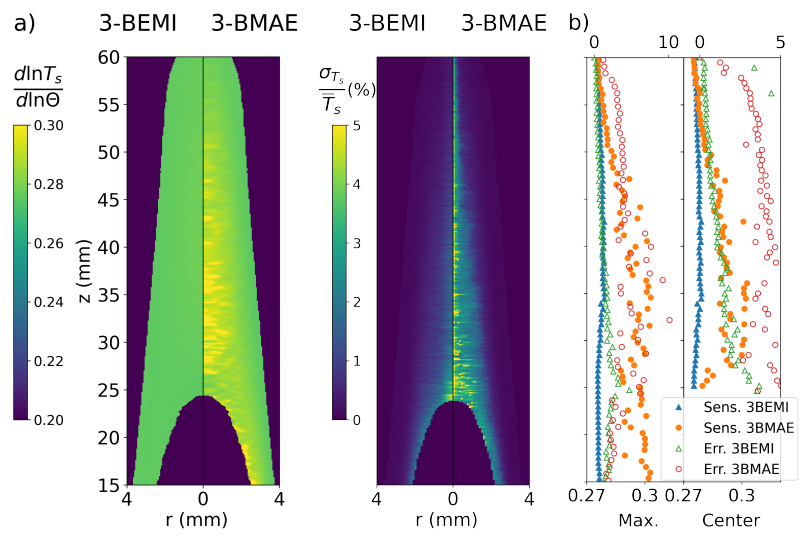

Fig. 4. (a) Fields of relative sensitivity (left) and error estimation by a MC approach (right) for the determination of $T_{S}$ with three-color based methodologies, and (b) maximum (left) and centerline (right) values of relative sensitivity (filled, bottom axis) and MC errors (unfilled, top axis).

centerline. Indeed, the smoothness $(s$, defined here as the mean absolute value of $\partial T / \partial z \times(T / z)$ ref with $(T / z)_{\text {ref }}$ a reference value) are 0.57 and 0.95 for 3-BEMI and 3-BMAE, respectively, where lower values represent a smoother curve. Similar trends are also obtained for $T_{S} 2$-BEMI and $T_{S}$ 2-BMAE methodologies ( $s=0.64$ and 1.0, respectively). Finally, the 3-BEMI approach is able to retrieve a wider high-temperature zone for $T_{S}$ than 2BEMI. In addition, $T_{s} 3$-BEMI presents slightly smoother profiles than $T_{s} 2$-BEMI ( $s=0.57$ and 0.64 , respectively). These behaviors are attributed to the increased spectral information captured by the RGB camera as compared with common 2-color techniques.

The sensitivity of $T_{S}$ for both three-color methods is quantified in terms of $\partial \ln T_{S} / \partial \ln \Theta_{S, H}$ (see Supplemental Document). For the error analysis based on a Monte Carlo (MC) approach, the propagation of uncertainties is estimated by considering a Gaussian dispersion of each measured signal ( $R, G$ and $B$ ) with a $0.5 \%$ standard deviation of the projected emission signals. Such value corresponds to $10 \%$ of the mean ratio between the standard deviation and the average of the fifty flame images $\left(S^{\prime}\right)$ at the blue band. This band was chosen because of its smallest signal-to-noise ratio that is associated to the most challenging detection in flames. The relative MC error is then quantified as the standard deviation divided by the mean $T_{S}\left(\sigma / \bar{T}_{s}\right)$ of 50 perturbed measured signals $\left(S_{i}^{\prime}\right)$ fields. These uncertainties are reported in Fig. 4a. The sensitivity of $T_{S}$ is nearly constant for the 3-BEMI technique $(\sim 0.27)$, being lower than the sensitivity of the 3-BMAE model on the whole flame, and specially toward the centerline. A similar behavior for relative errors based on the MC approach can also be observed in Fig. 4a. Consistently, errors are accumulated toward the centerline. These results can also be noted in the profiles of maximum and centerline values of sensitivities (bottom axis) and MC errors (top axis) plotted in Fig. $4 \mathrm{~b}$. Based on these results, the 3-BEMI technique proved to be a robust technique for retrieving $T_{S}$ from measurements with an RGB camera without the need of intricate optical diagnostics.

\section{CONCLUSION}

This letter reported a three-color pyrometry method for soot temperature measurements in axisymmetric flames. The proposed methods (3-BEMI and 3-BMAE) specify a single expression to obtain the temperature using the total spectral captured emission of the three-color broadbands ( $R, G$ and $B$ ) by a CMOS CCD camera. The performance of the three-color procedure is validated numerically using simulated flames provided by the CoFlame code, where we observe that $T_{S}$ is recovered perfectly from the synthetic broadband images for the 3-BMAE technique, whereas slight discrepancies are observed for the 3-BEMI model because it neglects the self-absorption term. Experiments using a canonical axisymmetric flame show that the different approaches lead to similar qualitative and quantitative behavior on the retrieved $T_{S}$ field, being smoother for three-color and BEMI than for twocolor and BMAE methodologies. The 3-BMAE method requires a complex optical setup, a tedious iterative process for retrieving $T_{S}$, and the error accumulation in the deconvolution negatively affects the temperature reconstruction around the flame centerline. In contrast, 3-BEMI requires a simpler setup than 3-BMAE, delivering similar temperature values with smoother temperature field toward the center of the flame, thus demonstrating the reliability of the optical technique proposed. Finally, the uncertainty analysis shows that $T_{S} 3$-BMAE is more sensible to changes on the ratio of measured signals, which is confirmed by a Monte Carlo approach for the propagation of uncertainties.

Funding. This work was supported by Chile's ANID projects FONDECYT/Regular 1191758 and Postdoctoral 3190860, FONDEF ID18I10236 and DGIIP UTFSM/PIIC. The authors also gratefully acknowledge the support provided by Brazil's CNPq, under Research Grants No. 140994/2013-0, 306069/2015-6 and 403904/2016-1 and by CAPES/PrInt 88881.310634/2018-01.

Disclosures. The authors declare no conflicts of interest.

See Supplemental Document for supporting content.

\section{REFERENCES}

1. W. Yan, S. Zheng, and H. Zhou, Appl. Therm. Eng. 124, 1014 (2017).

2. P. B. Kuhn, B. Ma, B. C. Connelly, M. D. Smooke, and M. B. Long, Proc. Combust. Inst. 33, 743 (2011).

3. N. J. Kempema and M. B. Long, Opt. Lett. 43, 1103 (2018).

4. J. A. H. Dreyer, R. I. Slavchov, E. J. Rees, J. Akroyd, M. Salamanca, S. Mosbach, and M. Kraft, Appl. Opt. 58, 2662 (2019).

5. J. J. Cruz, L. F. F. da Silva, F. Escudero, F. Cepeda, J. C. Elicer-Cortés, and A. Fuentes, Combust. Sci. Technol. 0, 1 (2020).

6. F. Liu, K. A. Thomson, and G. J. Smallwood, Combust. Flame 160, 1693 (2013).

7. F. Liu, J. Yon, A. Fuentes, P. Lobo, G. J. Smallwood, and J. C. Corbin, Aerosol Sci. Technol. 54, 33 (2020).

8. G. Legros, Q. Wang, J. Bonnety, M. Kashif, C. Morin, J.-L. Consalvi, and F. Liu, Combust. Flame 162, 2705 (2015).

9. F. Escudero, A. Fuentes, J.-L. Consalvi, F. Liu, and R. Demarco, Fuel 183, 668 (2016).

10. A. Guibaud, J. Citerne, J. Orlac'h, O. Fujita, J.-L. Consalvi, J. Torero, and G. Legros, Proc. Combust. Inst. 37, 3959 (2019).

11. T. Panagiotou, Y. Levendis, and M. Delichatsios, Combust. Flame 104, 272 (1996).

12. R. Khatami and Y. A. Levendis., Combust. Flame 158, 1822 (2011).

13. N. Eaves, Q. Zhang, F. Liu, H. Guo, S. Dworkin, and M. Thomson, Comput. Phys. Commun. 207, 464 (2016).

14. J. Yon, R. Lemaire, E. Therssen, P. Desgroux, A. Coppalle, and K. Ren, Appl. Phys. B 104, 253 (2011).

15. A. Rodríguez, F. Escudero, J. Cruz, G. Carvajal, and A. Fuentes, Fuel 285, 119011 (2021).

16. W. Yan, D. Chen, Z. Yang, E. Yan, and P. Zhao, Energies 10, 750 (2017).

17. K. J. Daun, K. A. Thomson, F. Liu, and G. J. Smallwood, Appl. optics 45, 4638 (2006).

18. R. Y. Rubinstein and D. P. Kroese, Simulation and the Monte Carlo method, vol. 10 (John Wiley \& Sons, 2016). 


\section{FULL REFERENCES}

1. W. Yan, S. Zheng, and H. Zhou, "Experiments investigation on 2d distribution of soot temperature and volume fraction by image processing of visible radiation," Appl. Therm. Eng. 124, 1014 - 1022 (2017).

2. P. B. Kuhn, B. Ma, B. C. Connelly, M. D. Smooke, and M. B. Long, "Soot and thin-filament pyrometry using a color digital camera," Proc. Combust. Inst. 33, 743-750 (2011).

3. N. J. Kempema and M. B. Long, "Effect of soot self-absorption on color-ratio pyrometry in laminar coflow diffusion flames," Opt. Lett. 43, 1103-1106 (2018).

4. J. A. H. Dreyer, R. I. Slavchov, E. J. Rees, J. Akroyd, M. Salamanca, S. Mosbach, and M. Kraft, "Improved methodology for performing the inverse abel transform of flame images for color ratio pyrometry," Appl. Opt. 58, 2662-2670 (2019).

5. J. J. Cruz, L. F. F. da Silva, F. Escudero, F. Cepeda, J. C. ElicerCortés, and A. Fuentes, "Soot pyrometry by emission measurements at different wavelengths in laminar axisymmetric flames," Combust. Sci. Technol. 0, 1-18 (2020).

6. F. Liu, K. A. Thomson, and G. J. Smallwood, "Soot temperature and volume fraction retrieval from spectrally resolved flame emission measurement in laminar axisymmetric coflow diffusion flames: Effect of self-absorption," Combust. Flame 160, 1693-1705 (2013).

7. F. Liu, J. Yon, A. Fuentes, P. Lobo, G. J. Smallwood, and J. C. Corbin, "Review of recent literature on the light absorption properties of black carbon: Refractive index, mass absorption cross section, and absorption function," Aerosol Sci. Technol. 54, 33-51 (2020).

8. G. Legros, Q. Wang, J. Bonnety, M. Kashif, C. Morin, J.-L. Consalvi, and F. Liu, "Simultaneous soot temperature and volume fraction measurements in axis-symmetric flames by a two-dimensional modulated absorption/emission technique," Combust. Flame 162, 2705-2719 (2015).

9. F. Escudero, A. Fuentes, J.-L. Consalvi, F. Liu, and R. Demarco, "Unified behavior of soot production and radiative heat transfer in ethylene, propane and butane axisymmetric laminar diffusion flames at different oxygen indices," Fuel 183, 668-679 (2016).

10. A. Guibaud, J. Citerne, J. Orlac'h, O. Fujita, J.-L. Consalvi, J. Torero, and G. Legros, "Broadband modulated absorption/emission technique to probe sooting flames: Implementation, validation, and limitations," Proc. Combust. Inst. 37, 3959-3966 (2019).

11. T. Panagiotou, Y. Levendis, and M. Delichatsios, "Measurements of particle flame temperatures using three-color optical pyrometry," Combust. Flame 104, 272-287 (1996).

12. R. Khatami and Y. A. Levendis., "On the deduction of single coal particle combustion temperature from three-color optical pyrometry," Combust. Flame 158, 1822 - 1836 (2011).

13. N. Eaves, Q. Zhang, F. Liu, H. Guo, S. Dworkin, and M. Thomson, "CoFlame: A refined and validated numerical algorithm for modeling sooting laminar coflow diffusion flames," Comput. Phys. Commun. 207, 464-477 (2016).

14. J. Yon, R. Lemaire, E. Therssen, P. Desgroux, A. Coppalle, and K. Ren, "Examination of wavelength dependent soot optical properties of diesel and diesel/rapeseed methyl ester mixture by extinction spectra analysis and lii measurements," Appl. Phys. B 104, 253-271 (2011).

15. A. Rodríguez, F. Escudero, J. Cruz, G. Carvajal, and A. Fuentes, "Retrieving soot volume fraction fields for laminar axisymmetric diffusion flames using convolutional neural networks," Fuel 285, 119011 (2021).

16. W. Yan, D. Chen, Z. Yang, E. Yan, and P. Zhao, "Measurement of soot volume fraction and temperature for oxygen-enriched ethylene combustion based on flame image processing," Energies 10, 750 (2017).

17. K. J. Daun, K. A. Thomson, F. Liu, and G. J. Smallwood, "Deconvolution of axisymmetric flame properties using tikhonov regularization," Appl. optics 45, 4638-4646 (2006).

18. R. Y. Rubinstein and D. P. Kroese, Simulation and the Monte Carlo method, vol. 10 (John Wiley \& Sons, 2016). 